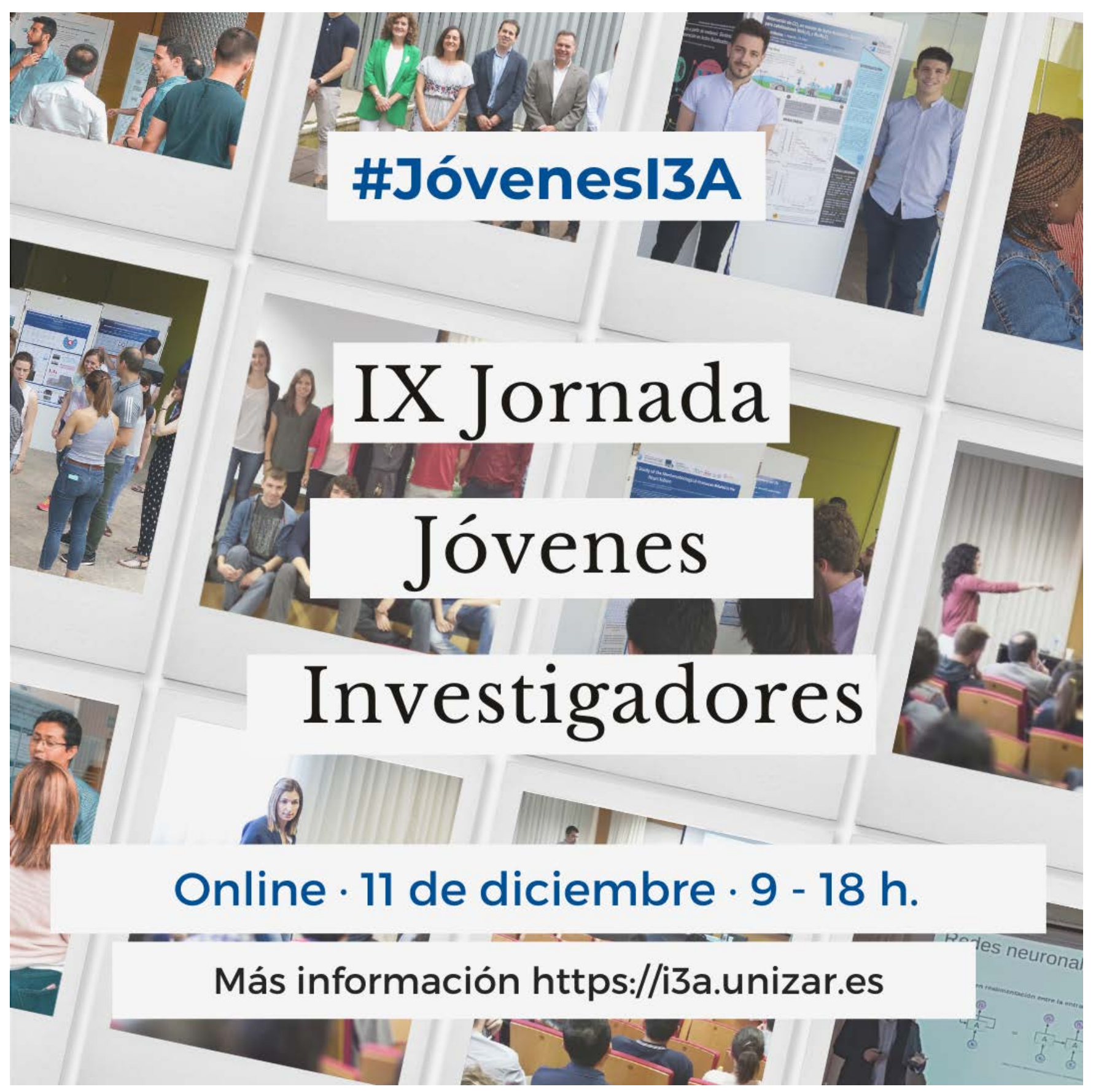




\section{JORNADA DE JÓVENES INVESTIGADORES DEL I3A}

Instituto de Investigación en Ingeniería de Aragón

Campus Río Ebro - Edificio I+D+i

c/ Mariano Esquillor $\mathrm{s} / \mathrm{n}$

50018 Zaragoza

i3a@unizar.es

$\begin{array}{ll}\text { Dirección editorial } & \text { José Jesús Guerrero } \\ \text { Consejo editorial } & \text { Pablo Laguna } \\ & \text { Elías Cueto } \\ & M^{a} \text { Ángeles Pérez } \\ & \text { José Ángel Peña } \\ & \text { Alfonso Ortega }\end{array}$




\section{PRESENTACIÓN}

Un año más el Instituto de Investigación en Ingeniería de Aragón (I3A) celebró su Jornada de Jóvenes Investigadores, punto de encuentro para aquellos que están realizando sus tesis doctorales en el I3A y una de las actividades con más proyección de las que se organizan en nuestro Instituto Universitario.

En esta ocasión el encuentro constituyó el eje principal de la I Jornada del I3A, un día dedicado a poner en valor el trabajo de investigación en nuestro instituto y en el que se entregó la "Distinción I3A" al grupo empresarial SAMCA por su mecenazgo y apoyo a la I+D+i del Instituto.

La Jornada de Jóvenes Investigadores, que alcanza ya su IX edición, reunió virtualmente a nuestros jóvenes investigadores e investigadoras con 49 estudios y líneas de trabajo presentadas en las áreas de Tecnologías de la Información y Comunicaciones, Ingeniería Biomédica, Tecnologías Industriales y Procesos y Reciclado.

El programa se complementó con tres conferencias científicas invitadas. En la conferencia inaugural, Manuel González Bedia, investigador del I3A y, actualmente, subdirector general de Actividad Universitaria Investigadora y vocal de la Comisión de Coordinación del Ministerio de Ciencia e Innovación y Ministerio de Universidades, hizo un recorrido por la política científica española y presentó la Estrategia de Inteligencia Artificial. Juan Domingo Tardós, investigador del grupo de Robótica, Percepción y Tiempo Real (RoPeRT), explicó a los más jóvenes "¿Cómo transferir el software resultado de tu investigación?" y Elías Cueto, subdirector del I3A e investigador del grupo de Mecánica Aplicada y Bioingeniería (AMB), intervino con la conferencia "Científicos artificiales: enseñando a los robots a hacer ciencia".

Todas las contribuciones recibidas a esta IX Jornada de Jóvenes Investigadores del I3A, además de los posters presentados, se recogen en este libro, que puede ser consultado también a través de la página web del Instituto (http://i3a.unizar.es)

Desde aquí, queremos dar las gracias a los jóvenes investigadores de nuestro Instituto que han participado y contribuido con su entusiasmo al desarrollo de este encuentro anual y a la I Jornada del I3A.

Pablo Laguna

Director del I3A
José J. Guerrero

Subdirector de formación 
JORNADA DEL I3A - IX JORNADA DE JÓVENES INVESTIGADORES - 11/12/2020

PROGRAMA

9:00 Inauguración Consejera de Ciencia, Universidad y Sociedad del Conocimiento, Maru Díaz; vicerrectora de Política Científica en funciones, Blanca Ros; director del I3A, Pablo Laguna

9:15 Entrega "Distinción I3A" al grupo empresarial SAMCA.

9:30 Conferencia inaugural

Manuel González Bedia Panorámica de la Política Científica en España

Modera: Pablo Laguna

10:30-11:00 Presentaciones orales (Modera Alfonso Ortega)

10:30 Conversión de $\mathrm{CO}_{2}$ en gas natural sintético: análisis de eficiencia mediante el uso de catalizadores basados en sepiolita

A Sanz-Martínez, J. Gurauskis, V. Gil, J. Herguido, J.A. Peña

10:45 Obtención de productos de valor añadido mediante la hidrogenólisis de glicerina sin aporte externo de hidrógeno

R. Raso, L. García, J. Ruiz, M. Oliva, J. Arauzo

11:00-12:00 Sesión de pósters (ANEXO) (Modera José J. Guerrero)

12:00-13:00 Presentaciones orales (Modera Ma Ángeles Pérez)

12:00 La capacidad del barorreflejo como índice de identificación de los pacientes de UCI preparados para el destete. P. Armañac, D. Hernando, J. Lázaro, C. de Haro, R. Magrans, L. Sarlabous, JF López-Aguilar, P. Laguna, E. Gil, L. Blanc, R. Bailón

12:15 Desarrollo e Implementación de Técnicas y Dispositivos para la Aplicación de Electroporación como Tratamiento Tumoral B. López-Alonso, H. Sarnago, O. Lucía y J.M. Burdío

12:30 Are the Cells Stronger than we Think? S. Hervás-Raluy, M.J. Gómez-Benito, C. Borau-Zamora, J.M.I García-Aznar

12:45 How to Predict the Long-term Course of Neurodegenerative Diseases? A. Montolío, J. Cegoñino, E. GarcíaMartín, A. Pérez del Palomar

13:00-14:00 Pausa Comida

14:30 Conferencia Juan Domingo Tardós ¿Cómo transferir el software resultado de tu investigación? Modera José J. Guerrero

15:30-16:30 Presentaciones orales (Modera José J. Guerrero)

15:30 Avances en la síntesis de alto nivel para la generación de hardware en FPGA: Modelos y programabilidad M.A. Dávila, R. Gran-Tejero, M. Villarroya y D. Suárez-Gracia

15:45 File-based race conditions in UNIX: TOCTOU R. Raducu, R.J. Rodríguez, P. Álvarez

16:00 Análisis computacional del cocinado de carne a la plancha. J. Moya, B. Calvo, J. Grasa

16:15 Radio over Fiber: An Alternative Broadband Network Technology for loT D. F. Paredes-Páliz, G. Royo, F. Aznar, C. Aldea, S. Celma

16:30 Conferencia Elías Cueto Científicos artificiales: enseñando a los robots a hacer ciencia Modera José Ángel Peña

17:30 Entrega de Premios

- Diplomas de becas y contratos Puente

- Premios a la Mejor contribución por división

* Ios títulos de las contribuciones contienen un hyperlink a los ficheros pdf del trabajo para facilitar su consulta. 


\section{SESIÓN DE PÓSTERS}

\section{INGENIERÍA BIOMÉDICA}

BI01. Numerical Approach of the Ciliary Muscle Contraction. A Preliminary Step to Reproduce Human Accommodation. I.Cabeza Gil, J. Grasa, A.Glasser, B. Calvo

BIO2. Estudio correlacional entre discapacidad cervical, kinesiofobia y consumo farmacológico en pacientes mayores de 65 años con cervicalgia crónica. J. Corral de Toro, C. Hidalgo García, J.M. Tricas Moreno, M.O. Lucha López, J. Rodríguez Sanz, C.J. Palacio Del Rio, L. Ferrández Laliena, S. Monti Ballano

BIO3. Estudio comparativo del Test de Flexión-Rotación y Rango de Movimiento de la Columna Cervical Superior en sujetos sanos y sujetos con dolor cervical con restricción de movilidad cervical superior. J. Corral de Toro, C. Hidalgo García, J.M. Tricas Moreno, M.O. Lucha López, J. Rodríguez Sanz, R. Sánchez Rodríguez, L. Ferrández Laliena, S. Monti Ballano

BI04. Análisis de marcadores de salud en el trail running. D. Fernández de Landa Nicolau, M.O. Lucha López, S. Monti Ballano, J. Corral de Toro, L. Ferrández Laliena, J.M. Tricas Moreno

BIO5. Efecto de un programa de ejercicio terapéutico en la mejora funcional y prevención de lesiones de rodilla en el fútbol sala femenino semiprofesional. L. Ferrández Laliena, R. Sánchez Rodríguez, L. Vicente Pina, M.O. Lucha López, S. Monti Ballano, J. Corral de Toro, D. Fernández de Landa Nicolau, J.M. Tricas Moreno

BI07. Crecimiento de placa de ateroma en arteria carótida de paciente específico según diferentes estímulos mecánicos. P. Hernández López, M. Cilla Hernández, M.A. Martínez, Barca, E. Peña Baquedano

BIO8. Biological Matrix for 2.5D Renal Model in vitro. A. Lacueva Aparicio, L. Fernández Ledesma, I. Giménez López

BI09. Estudio de la viabilidad de la segmentación de placas de ateroma vulnerables en coronarias a partir del campo de desplazamientos compatibles con imágenes IVUS. Á. T. Latorre, E. Peña, M.A. Martínez, M. Cilla

BI010. Range of Motion of the Upper Cervical Spine: flexion, extension, lateral bending, and axial rotation. A.I. Lorente, C. Hidalgo García, J. Rodríguez Sanz, M. Maza Frechín, A. Pérez Bellmunt

BI011. Influencia del envejecimiento en la función oculomotora. Comparación entre ancianos con cefalea y ancianos asintomáticos. S. Monti Ballano, M. Malo Urriés, M.O. Lucha López, J.M. Tricas Moreno, J. Corral de Toro, S. Márquez Gonzalvo

BI012. Modelo de simulación del crecimiento de callo óseo en consolidación de fracturas. J.M. Naveiro Gómez, J. Rosell Pradas

BI013. Dinámica periódica de la repolarización como predictor de muerte súbita en pacientes con insuficiencia cardíaca crónica. S. Palacios, I. Cygankiewicz, A. Bayés-de-Luna, E. Pueyo, J.P.Martínez

BI014. Alginate-based Microcapsules for Cell Therapy: A Combination of Techniques Designed to Characterize their Stiffness and Surface Properties. L. Paz Artigas, M. Virumbrales-Muñoz, J. Ciriza, C. Alcaine, A. Espona, M. Doblaré, L. Sáenz del Burgo; K. Ziani, J.L. Pedraz, L. Fernández, I. Ochoa

BI015. De la prueba de esfuerzo a la predicción de muerte súbita cardiaca usando marcadores no invasivos. C. Pérez, E. Pueyo, J.P. Martínez, P. Laguna 
BI016. Epigenética computacional: simulación de mecanismos de adquisición de resistencia a fármacos en cultivos celulares. M. Pérez-Aliacar, J. Ayensa-Jiménez, M. H. Doweidar, M. Doblaré

BI017. Experimental Study of the Dissection Properties of Porcine Aorta. I. Ríos Ruiz, M.A. Martínez Barca, E. Peña Baquedano

BI018. Análisis biomecánico de fracturas subtrocantéreas femorales. Factores críticos para una adecuada consolidación. J. Rosell Pradas, J.M. Naveiro Gómez, C. Martínez Aznar

BIO 19. Finite Element Model for Cardiac Cell mechano-electrical stimulation. P. Urdeitx Diaz, M. H. Doweidar

\section{PROCESOS Y RECICLADO}

PyR1. Producción de bioetanol carburante a partir del residuo mucilaginoso de cacao CCN-51 y su simulación en Aspen Plus ${ }^{\circledR}$. J. Delgado, J. Soler, J.A. Peña

PyR2. Ammonia Oxidation at High Pressure as a Carbon Free Fuel. P. García Ruiz, M. Abián, U. Alzueta

PyR3. Identification of Polyamide 6 and Polyamide 66 Oligomers Using lon Mobility Time-of-flight Mass Spectrometry and Risk Assessment Study of Their Migration from Kitchenware to Foodstuff. X. Song, E. Canellas, C. Nerín

\section{TECNOLOGÍAS INDUSTRIALES}

TI1. Redes neuronales guiadas con variables internas físicas: cuando la física guía a los datos en el Aprendizaje Profundo. J. Ayensa-Jiménez, M. H. Doweidar, M. Doblaré

T12. Implementación de redes neuronales en FPGAs usando tipos de datos de punto fijo. . Enériz, N. Medrano, B. Calvo

T13. Technical and Operational Feasibility, for the business analysis, of running an Autopista Ferroviaria with Iberian track gauge between Port of Algeciras and Zaragoza-Plaza. J. Escobar de la Iglesia, E. Larrodé Pellicer

T14. Hacia un científico digital. Q. Hernández, A. Badías, D. González, F. Chinesta, E. Cueto

TI5. Estrategia de Diseño de Amplificadores de Bajo Ruido Integrados en Tecnología CMOS nanométrica. A. D. Martínez Pérez, P. A. Martínez Martínez, G. Royo, F. Aznar, S. Celma

TI6 Labour Productivity in Mixed-model Manual Assembly Changeovers. A. Miqueo, M. Torralba, J,A, Yagüe Fabra

T17. Data Learning of Fluid Dynamics for Physically Informed Digital Twins. B. Moya, I. Alfaro, D. González, F. Chinesta, E. Cueto

\section{TECNOLOGIAS DE LA INFORMACIÓN Y LA COMUNICACIÓN}

TICs1. Omnidirectional Image Data-set for Computer Vision Applications. B. Berenguel-Baeta, J. Bermudez-Cameo, J. J. Guerrero

TICs2. Etapas electrónicas de potencia resonantes multi-salida para calentamiento por inducción doméstico. P. Guillén, H. Sarnago, Ó. Lucía y J.M. Burdío

TICs3. Integración de tecnologías de apoyo para educación especial. M.A. Guillomía, J. Falcó Boudet, J.I. Artigas

TICs4. Towards Improving Agriculture Sustainability through Multifactorial Machine Learning. F.J. Lacueva Pérez, R. del Hoyo, S. Ilarri Artigas 
TICs5. Fully Charged: Characterizing the Data Requirements of Services and Apps to Optimize the Use of Energy. J.A. Larracoechea, P. Roose, S. Ilarri

TICs6. Imperceptible Manipulation of Lateral Camera Motion for Improved Virtual Reality Applications. A. Serrano, D. Martin, D. Gutiérrez, K. Myszkowski, B. Masiá

TICs7. Increasing Sensitivity of Optical Receivers using a Divide-and-Conquer Technique. G. Royo, A.D. Martínez-Pérez, C. Aldea Chagoyen, S. Celma

TICs8. Influence of Field of View in Visual Prostheses Design: Analysis with a VR System. M. Sánchez-García, R. Martínez-Cantín, J. Bermúdez-Cameo, J. J. Guerrero

TICs9. Automatic Feature Extraction from Biosignals Using Convolutional Neural Models. J. Torres, J.D. Buldain

TICs10. Data Exfiltration in loT Protocols. D. Uroz, R. J. Rodríguez

TICs11. Timbre Comparison in Note Tracking from Onset, Frames and Pitch Estimation. C. Hernández-Oliván, I. Zay Pinilla, J. R. Beltran 